\title{
INTENSITY AND POLARISATION BISTABILITY IN TWO PRIMARY-WAVE MIXING IN MEDIA WITH SECOND-ORDER NONLINEARITY
}

\author{
M. SERoczyńska AND A. Zagórski \\ Faculty of Physics, Warsaw University of Technology \\ Koszykowa 75, 00-662 Warszawa, Poland
}

(Received March 26, 2001)

\begin{abstract}
In this paper we analyse theoretically the transmission of two strong laser beams through a medium exhibiting optical nonlinearity of the second order combined with a linear anisotropy. A standard model of two wave mixing was applied and closed formulas for intensity and polarisation state were obtained. Numerical calculations show that outgoing beams are very sensitive to the state of incoming beams and to the material characteristics. Under some realistic conditions we were able to obtain bistability of both intensity and polarisation state. The results also show how to modulate the intensity and polarisation state of one wave by another one.

PACS numbers: $42.65 . \mathrm{Hw}, 42.65 . \mathrm{Ky}$
\end{abstract}

\section{Introduction}

There is a number of effects resulting from the three-wave interaction via nonlinear media of second order. Among them the optical bistability belongs to most spectacular ones. This phenomenon may be attributed both to intensity and to polarisation state of the wave. Both cases were intensively investigated since years as their potential applications seemed to be very wide (see [1-3] for a review). In media exhibiting third-order nonlinearity this effect is pretty well understood and described, whereas in second-order media (for instance a variety of organic materials) the theory of bistability is much less developed. Additional difficulty arises from anisotropy (example of material exhibiting anisotropy as well as nonlinearity of second order is $\mathrm{LiJO}_{3}$ ). In this paper we take into account both properties.

Optical bistability is usually observed by allowing a probe laser beam transmit a plate. The outgoing beam depends on the incoming one in a manner typical of materials exhibiting hysteresis. Much more complicated is the bistability arising from two-wave interaction when both waves have comparable intensities. The 
problem is sufficiently well solved for third-order media [4]. In this paper we concentrate attention on processes leading to second-order bistability. Following the method developed in [5] we have derived analytical formulas for intensities and polarisation describing parameters and then performed numerical calculations.

\section{Physical model and equations of motion}

The linear anisotropy of any material is described by the electric permittivity tensor $\varepsilon$. Its form depends on the choice of coordinate axes. If the $O z$ axis coincides with the main axis of the symmetry of the material, then

$$
\varepsilon=\left[\begin{array}{ccc}
\varepsilon^{(0)} & 0 & 0 \\
0 & \varepsilon^{(0)} & 0 \\
0 & 0 & \varepsilon^{(e)}
\end{array}\right]
$$

The nonlinear susceptibility $\chi$ is a tensor of the third rank, which for $\mathrm{LiJO}_{3}$ has only 7 different from zero components. Six of them are the same, one has another value

$$
\chi_{133}=\chi_{223}=\chi_{232}=\chi_{131}=\chi_{311}=\chi_{322} \equiv b, \quad \chi_{333}=a .
$$

Approximate values of these two parameters for this material are $b=$ $-96.5 \times 10^{-15} \mathrm{C} / \mathrm{V}^{2}$ and $a=-74.2 \times 10^{-15} \mathrm{C} / \mathrm{V}^{2}$.

Now we assume that two beams of the frequencies $\omega_{1}$ (let it be $3.67 \times 10^{15} \mathrm{~Hz}$ ) and $\omega_{2}\left(300 \times 10^{15} \mathrm{~Hz}\right)$ fall on the plate made of the above-mentioned material. Their electric vectors are $\boldsymbol{E}_{1}$ and $\boldsymbol{E}_{2}$, respectively; both are of the order $300 \mathrm{~V} / \mathrm{m}$. Inside the plate each vector may be decomposed into two components: perpendicular (index p) and parallel (index r) to the incident plane: $\boldsymbol{E}=\boldsymbol{e}^{\mathrm{p}} E^{\mathrm{p}}+\boldsymbol{e}^{\mathrm{r}} E^{\mathrm{r}}$. The versor $e^{\mathrm{p}}=e_{y}(O y$ axis is perpendicular to the incident plane), whereas $\boldsymbol{e}^{\mathrm{r}}=\alpha \boldsymbol{e}_{x}+\beta \boldsymbol{e}_{z}$, where $\alpha$ and $\beta$ determine the angle between the $\boldsymbol{e}^{\mathrm{r}}$ and the main axis of the crystal $\left(\alpha^{2}+\beta^{2}=1\right)$.

In the same way one can decompose the electrical vector $\boldsymbol{E}$ of all waves generated in the process of nonlinear interactions of the second order of the frequencies: $2 \omega_{1}, 2 \omega_{2}, \omega \pm \omega_{2}$. In each component we may separate the complex amplitude $A$ and the phase factor according to the formula

$$
E=A \mathrm{e}^{-\mathrm{i} k r+\mathrm{i} \omega t}
$$

This formula applies independently to both types of polarisation ( $p$ and $r$ ).

Non-vanishing elements of the nonlinear electrical susceptibility tensor in the framework connected with $e^{\mathrm{P}}$ and $e^{\mathrm{r}}$ are

$$
\begin{aligned}
& \boldsymbol{e}^{\mathrm{p}} \chi \boldsymbol{e}^{\mathrm{p}} \boldsymbol{e}^{\mathrm{r}}=b \beta, \quad \boldsymbol{e}^{\mathrm{p}} \chi \boldsymbol{e}^{\mathrm{r}} \boldsymbol{e}^{\mathrm{p}}=b \beta, \quad \boldsymbol{e}^{\mathrm{r}} \chi \boldsymbol{e}^{\mathrm{p}} \boldsymbol{e}^{\mathrm{p}}=b \beta, \\
& \boldsymbol{e}^{\mathrm{r}} \chi \boldsymbol{e}^{\mathrm{p}} \boldsymbol{e}^{\mathrm{r}}=b(2 \alpha+\beta) \alpha \beta .
\end{aligned}
$$

The nonlinear polarisation $P$ can be expressed as a sum of components $P^{\mathrm{p}}$ and $P^{\mathrm{r}}$ for each frequency. For the sake of simplicity we adopt the following rule for the index $j$ (always the lower one) of particular frequencies:

$$
\begin{array}{ccccccc}
\text { frequency } & \omega_{1} & \omega_{2} & 2 \omega_{1} & 2 \omega_{2} & \omega_{1}-\omega_{2} & \omega_{1}+\omega_{2} \\
j & 1 & 2 & 3 & 4 & 5 & 6
\end{array} .
$$


These components may be calculated analytically (we omit here the details). At the end we get the following formulae:

$$
\begin{aligned}
& P_{1}^{\mathrm{p}}=b \beta\left[A_{6}^{\mathrm{p}} \bar{A}_{2}^{\mathrm{r}} \mathrm{e}^{-\mathrm{i}\left(\boldsymbol{k}_{6}^{\mathrm{P}}-\mathbb{k}_{2}^{\mathrm{r}}\right) \boldsymbol{r}}+A_{6}^{\mathrm{r}} \bar{A}_{2}^{\mathrm{p}} \mathrm{e}^{\mathrm{i}\left(\boldsymbol{k}_{2}^{\mathrm{p}}-\boldsymbol{k}_{6}^{\mathrm{r}}\right) \boldsymbol{r}}+A_{3}^{\mathrm{p}} \bar{A}_{1}^{\mathrm{r}} \mathrm{e}^{-\mathrm{i}\left(\boldsymbol{k}_{3}^{\mathrm{p}}-\boldsymbol{k}_{1}^{\mathrm{r}}\right) \boldsymbol{r}}\right. \\
& \left.+A_{3}^{\mathrm{r}} \bar{A}_{1}^{\mathrm{p}} \mathrm{e}^{-\mathrm{i}\left(\boldsymbol{k}_{3}^{\mathrm{r}}-\boldsymbol{k}_{1}^{\mathrm{p}}\right) \boldsymbol{r}}+A_{5}^{\mathrm{p}} A_{2}^{\mathrm{r}} \mathrm{e}^{-\mathrm{i}\left(\boldsymbol{k}_{5}^{\mathrm{p}}+\boldsymbol{k}_{2}^{\mathrm{r}}\right) \boldsymbol{r}}+A_{5}^{\mathrm{r}} A_{2}^{\mathrm{p}} \mathrm{e}^{-\mathrm{i}\left(\boldsymbol{k}_{5}^{\mathrm{r}}+\boldsymbol{k}_{2}^{\mathrm{p}}\right) \boldsymbol{r}}\right], \\
& P_{1}^{\mathrm{r}}=b \beta\left[A_{6}^{\mathrm{p}} \bar{A}_{2}^{\mathrm{r}} \mathrm{e}^{-\mathrm{i}\left(\boldsymbol{k}_{6}^{\mathrm{p}}-\boldsymbol{k}_{2}^{\mathrm{r}}\right) \boldsymbol{r}}+A_{5}^{\mathrm{p}} A_{2}^{\mathrm{P}} \mathrm{e}^{-\mathrm{i}\left(\boldsymbol{k}_{5}^{\mathrm{p}}+\boldsymbol{k}_{2}^{\mathrm{p}}\right) \boldsymbol{r}}+A_{3}^{\mathrm{p}} \bar{A}_{1}^{\mathrm{p}} \mathrm{e}^{-\mathrm{i}\left(\boldsymbol{k}_{5}^{\mathrm{p}}-\boldsymbol{k}_{1}^{\mathrm{p}}\right) \boldsymbol{r}}\right] \\
& +b \beta \alpha(2 \alpha+\beta)\left[A_{6}^{\mathrm{r}} \bar{A}_{2}^{\mathrm{r}} \mathrm{e}^{-\mathrm{i}\left(\boldsymbol{k}_{6}^{\mathrm{r}}-\mathbb{k}_{2}^{\mathrm{r}}\right) \boldsymbol{r}}+A_{3}^{\mathrm{r}} \bar{A}_{1}^{\mathrm{r}} \mathrm{e}^{-\mathrm{i}\left(\boldsymbol{Z}_{3}^{\mathrm{r}}-\mathbb{k}_{1}^{\mathrm{r}}\right) \boldsymbol{r}}+A_{2}^{\mathrm{r}} A_{5}^{\mathrm{r}} \mathrm{e}^{-\mathrm{i}\left(\boldsymbol{R}_{2}^{\mathrm{r}}+\boldsymbol{k}_{5}^{\mathrm{r}}\right) \boldsymbol{r}}\right], \\
& P_{2}^{\mathrm{p}}=b \beta\left[A_{6}^{\mathrm{P}} \bar{A}_{1}^{\mathrm{r}} \mathrm{e}^{-\mathrm{i}\left(\boldsymbol{k}_{6}^{\mathrm{p}}-\mathbb{R}_{1}^{\mathrm{r}}\right) \boldsymbol{r}}+A_{6}^{\mathrm{r}} \bar{A}_{1}^{\mathrm{p}} \mathrm{e}^{\mathrm{i}\left(\boldsymbol{k}_{1}^{\mathrm{p}}-\boldsymbol{k}_{6}^{\mathrm{r}}\right) \boldsymbol{r}}+A_{4}^{\mathrm{p}} \bar{A}_{2}^{\mathrm{r}} \mathrm{e}^{-\mathrm{i}\left(\boldsymbol{k}_{4}^{\mathrm{p}}-\boldsymbol{k}_{2}^{\mathrm{r}}\right) \boldsymbol{r}}\right. \\
& \left.+A_{4}^{\mathrm{r}} \bar{A}_{2}^{\mathrm{p}} \mathrm{e}^{-\mathrm{i}\left(\boldsymbol{k}_{4}^{\mathrm{r}}-\boldsymbol{k}_{2}^{\mathrm{p}}\right) \boldsymbol{r}}+A_{1}^{\mathrm{p}} \bar{A}_{5}^{\mathrm{r}} \mathrm{e}^{-\mathrm{i}\left(\boldsymbol{k}_{1}^{\mathrm{p}}-\boldsymbol{k}_{5}^{\mathrm{r}}\right) \boldsymbol{r}}+A_{1}^{\mathrm{r}} \bar{A}_{5}^{\mathrm{p}} \mathrm{e}^{-\mathrm{i}\left(\boldsymbol{\pi}_{1}^{\mathrm{r}}-\boldsymbol{k}_{5}^{\mathrm{p}}\right) \boldsymbol{r}}\right], \\
& P_{2}^{\mathrm{r}}=b \beta\left[A_{6}^{\mathrm{p}} \bar{A}_{1}^{\mathrm{r}} \mathrm{e}^{-\mathrm{i}\left(\boldsymbol{k}_{6}^{\mathrm{p}}-\boldsymbol{k}_{1}^{\mathrm{r}}\right) \pi}+A_{1}^{\mathrm{p}} \bar{A}_{5}^{\mathrm{p}} \mathrm{e}^{-\mathrm{i}\left(\boldsymbol{k}_{1}^{\mathrm{p}}-\boldsymbol{k}_{5}^{\mathrm{p}}\right) \boldsymbol{r}}+A_{4}^{\mathrm{p}} \bar{A}_{2}^{\mathrm{p}} \mathrm{e}^{-\mathrm{i}\left(\boldsymbol{k}_{4}^{\mathrm{p}}-\boldsymbol{k}_{2}^{\mathrm{p}}\right) \boldsymbol{r}}\right] \\
& +b \beta \alpha(2 \alpha+\beta)\left[A_{6}^{\mathrm{r}} \bar{A}_{1}^{\mathrm{r}} \mathrm{e}^{-\mathrm{i}\left(\boldsymbol{k}_{6}^{\mathrm{r}}-\mathbb{k}_{1}^{\mathrm{r}}\right) \boldsymbol{r}}+A_{1}^{\mathrm{r}} \bar{A}_{5}^{\mathrm{r}} \mathrm{e}^{-\mathrm{i}\left(\boldsymbol{k}_{1}^{\mathrm{r}}-\mathbb{k}_{5}^{\mathrm{r}}\right) \boldsymbol{r}}+A_{4}^{\mathrm{r}} \bar{A}_{2}^{\mathrm{r}} \mathrm{e}^{-\mathrm{i}\left(\boldsymbol{k}_{4}^{\mathrm{p}}-\boldsymbol{k}_{2}^{\mathrm{r}}\right) \boldsymbol{r}}\right], \\
& P_{3}^{\mathrm{p}}=b \beta\left[A_{1}^{\mathrm{p}} A_{1}^{\mathrm{r}} \mathrm{e}^{-\mathrm{i}\left(\boldsymbol{k}_{1}^{\mathrm{P}}+\not_{1}^{\mathrm{r}}\right) r}+A_{1}^{\mathrm{r}} A_{1}^{\mathrm{p}} \mathrm{e}^{-\mathrm{i}\left(\mathbb{R}_{1}^{\mathrm{P}}+\mathbb{k}_{1}^{\mathrm{r}}\right) r}\right], \\
& P_{3}^{\mathrm{r}}=b \beta A_{1}^{\mathrm{P}} A_{1}^{\mathrm{p}} \mathrm{e}^{-2 \mathrm{i} k_{1}^{\mathrm{P}} r}+b \beta(2 \alpha+\beta) \alpha A_{1}^{\mathrm{r}} A_{1}^{\mathrm{r}} \mathrm{e}^{-2 \mathrm{i} k_{1}^{\mathrm{r}} \pi} \\
& P_{4}^{\mathrm{p}}=b \beta\left[A_{2}^{\mathrm{P}} A_{2}^{\mathrm{r}} \mathrm{e}^{-\mathrm{i}\left(\mathbb{k}_{2}^{\mathrm{p}}+\mathbb{k}_{2}^{\mathrm{r}}\right) r}+A_{2}^{\mathrm{r}} A_{2}^{\mathrm{p}} \mathrm{e}^{-\mathrm{i}\left(\mathbb{k}_{2}^{\mathrm{p}}+\mathbb{K}_{2}^{\mathrm{r}}\right) \pi}\right], \\
& P_{4}^{\mathrm{r}}=b \beta A_{2}^{\mathrm{p}} A_{2}^{\mathrm{p}} \mathrm{e}^{-2 \mathrm{i} k_{2}^{\mathrm{P}} r}+b \beta(2 \alpha+\beta) \alpha A_{2}^{\mathrm{r}} A_{2}^{\mathrm{r}} \mathrm{e}^{-2 \mathrm{i} k_{2}^{\mathrm{r}} \pi} \\
& P_{5}^{\mathrm{p}}=b \beta\left[A_{1}^{\mathrm{p}} A_{2}^{\mathrm{r}} \mathrm{e}^{-\mathrm{i}\left(\mathbb{k}_{1}^{\mathrm{p}}-\mathbb{R}_{2}^{\mathrm{r}}\right) \boldsymbol{r}}+A_{1}^{\mathrm{r}} \bar{A}_{2}^{\mathrm{p}} \mathrm{e}^{-\mathrm{i}\left(k_{1}^{\mathrm{r}}-\mathbb{k}_{2}^{\mathrm{p}}\right) \boldsymbol{r}}\right], \\
& P_{5}^{\mathrm{r}}=b \beta A_{1}^{\mathrm{p}} \bar{A}_{2}^{\mathrm{p}} \mathrm{e}^{-\mathrm{i}\left(\mathbb{k}_{1}^{\mathrm{p}}-\mathbb{R}_{2}^{\mathrm{p}}\right) \boldsymbol{r}}+b \beta(2 \alpha+\beta) \alpha A_{1}^{\mathrm{r}} \bar{A}_{2}^{\mathrm{r}} \mathrm{e}^{-\mathrm{i}\left(\boldsymbol{k}_{1}^{\mathrm{r}}-\boldsymbol{k}_{2}^{\mathrm{r}}\right) \boldsymbol{r}} \text {, } \\
& P_{6}^{\mathrm{p}}=b \beta\left[A_{1}^{\mathrm{P}} A_{2}^{\mathrm{r}} \mathrm{e}^{-\mathrm{i}\left(\mathbb{k}_{1}^{\mathrm{p}}+\mathbb{k}_{2}^{\mathrm{r}}\right) r}+A_{1}^{\mathrm{r}} A_{2}^{\mathrm{P}} \mathrm{e}^{-\mathrm{i}\left(\mathbb{k}_{1}^{\mathrm{r}}+\mathbb{k}_{2}^{\mathrm{p}}\right) r}\right], \\
& P_{6}^{\mathrm{r}}=b \beta A_{1}^{\mathrm{p}} A_{2}^{\mathrm{p}} \mathrm{e}^{-\mathrm{i}\left(\boldsymbol{k}_{1}^{\mathrm{p}}+\mathbb{k}_{2}^{\mathrm{p}}\right) r}+b \beta(2 \alpha+\beta) \alpha A_{1}^{\mathrm{r}} A_{2}^{\mathrm{r}} \mathrm{e}^{-\mathrm{i}\left(\mathbb{k}_{1}^{\mathrm{r}}+\mathbb{k}_{2}^{\mathrm{r}}\right) r} .
\end{aligned}
$$

Equations (5)-(10) are sufficient for writing exact formulae for amplitudes $A_{j}$ of the six waves considered. In the slowly-varying amplitude approximation it is possible to write explicit formulae for the first derivatives of these variables with respect to the space coordinate $s$ ( $s$ is measured along the $O z$ axis, parallel to the propagation vector $\boldsymbol{k}$ ). For instance, the $A_{1}^{\mathrm{p}}$ fulfils - in the lack of absorption the equation 


$$
\begin{aligned}
\frac{\mathrm{d} A_{1}^{\mathrm{P}}}{\mathrm{d} s} & =-\mathrm{i} \frac{\omega_{1}^{2}}{2 k_{1}^{\mathrm{p}}} \mu_{0} b \beta\left[A_{6}^{\mathrm{p}} \bar{A}_{2}^{\mathrm{r}} \mathrm{e}^{-\mathrm{i}\left(k_{6}^{\mathrm{p}}-k_{2}^{\mathrm{r}}\right) s}+A_{6}^{\mathrm{r}} \bar{A}_{2}^{\mathrm{p}} \mathrm{e}^{-\mathrm{i}\left(k_{6}^{\mathrm{r}}-k_{2}^{\mathrm{p}}\right) s}+A_{3}^{\mathrm{p}} \bar{A}_{1}^{\mathrm{r}} \mathrm{e}^{-\mathrm{i}\left(k_{3}^{\mathrm{p}}-k_{1}^{\mathrm{r}}\right) s}\right. \\
& \left.+A_{5}^{\mathrm{p}} A_{2}^{\mathrm{r}} \mathrm{e}^{-\mathrm{i}\left(k_{5}^{\mathrm{p}}+k_{2}^{\mathrm{r}}\right) s}+A_{5}^{\mathrm{r}} A_{2}^{\mathrm{p}} \mathrm{e}^{-\mathrm{i}\left(k_{5}^{\mathrm{r}}+k_{2}^{\mathrm{p}}\right) s}+A_{3}^{\mathrm{r}} \bar{A}_{1}^{\mathrm{p}} \mathrm{e}^{-\mathrm{i}\left(k_{3}^{\mathrm{r}}-k_{1}^{\mathrm{p}}\right) s}\right] .
\end{aligned}
$$

Similar formulae hold for the remaining eleven components of $\boldsymbol{P}$.

Successive differentiation with respect to $s$ leads to a new set of equations, in which we neglect terms containing rapidly varying phase factors. In this way from Eq. (11) we get

$$
\begin{aligned}
\frac{\mathrm{d}^{2} A_{1}^{\mathrm{p}}}{\mathrm{d} s^{2}} & =-\frac{\omega_{1}^{2}}{2 k_{1}^{\mathrm{p}}}\left(\mu_{0} b \beta\right)^{2}\left[\frac{\omega_{6}^{2}}{2 k_{6}^{\mathrm{p}}}\left|A_{1}^{\mathrm{r}}\right|^{2}+\frac{\omega_{6}^{2}}{2 k_{6}^{\mathrm{r}}}\left|A_{2}^{\mathrm{P}}\right|^{2}+\frac{4 \omega_{1}^{2}}{k_{3}^{\mathrm{p}}}\left|A_{1}^{\mathrm{r}}\right|^{2}\right. \\
& \left.+\frac{\omega_{5}^{2}}{2 k_{5}^{\mathrm{p}}}\left|A_{2}^{\mathrm{r}}\right|^{2}+\frac{2 \omega_{1}^{2}}{k_{6}^{\mathrm{r}}}\left|A_{1}^{\mathrm{p}}\right|^{2}\right] A_{1}^{\mathrm{p}} .
\end{aligned}
$$

Analogous equations describe other amplitudes. The most important now is that the expression in the bracket [...] includes only terms proportional to the module $|A|$ of the complex amplitudes $A$, which in turn are directly proportional to corresponding intensities. Usually, one may assume that intensities of waves participating in the nonlinear interaction do not change essentially; much more important are changes of phases $(\Phi)$ of the waves. They may be described as follows. Making use of the notation

$$
A=|A| \mathrm{e}^{\mathrm{i} \Phi}
$$

and of the condition

$$
|A|=\text { const }
$$

we get the equation: $A^{\prime \prime}=\left[-\left(\Phi^{\prime}\right)^{2}+\mathrm{i} \Phi^{\prime \prime}\right]|A| \mathrm{e}^{\mathrm{i} \Phi}$. Neglecting second derivatives and using the above approximations, we obtain from (12) the following equation:

$$
\frac{\mathrm{d} \Phi_{j}^{\alpha}}{\mathrm{d} s}=\sqrt{\sum_{l, \xi} \Gamma_{j l}^{\alpha \xi}\left|A_{l}^{\xi}\right|^{2}} \equiv \eta_{j}^{\alpha},
$$

where the coefficients $\Gamma$ make a set of 16 numbers, which may be divided into 4 matrices of the rank $2 \times 2$ with respect to lower (frequency) indexes. They have the following form:

$$
\begin{aligned}
& {\left[\Gamma^{\mathrm{pp}}\right]=\mu_{0}^{2} b^{2} \beta^{2}\left[\begin{array}{cc}
\frac{\omega_{1}^{2} \omega_{6}^{2}}{4 k_{1}^{\mathrm{p}} k_{6}^{\mathrm{r}}}, & \frac{\omega_{1}^{2} \omega_{6}^{2}}{4 k_{1}^{\mathrm{p}} k_{6}^{\mathrm{r}}} \\
\frac{\omega_{1}^{2} \omega_{6}^{2}}{4 k_{1}^{\mathrm{p}} k_{6}^{\mathrm{r}}}, & \frac{\omega_{2}^{4}}{k_{2}^{\mathrm{p}} k_{4}^{\mathrm{r}}}
\end{array}\right],} \\
& {\left[\Gamma^{\mathrm{pr}}\right]=\mu_{0}^{2} b^{2} \beta^{2}\left[\begin{array}{cc}
\frac{\omega_{1}^{2} \omega_{6}^{2}}{4 k_{1}^{\mathrm{P}} k_{6}^{\mathrm{r}}}+\frac{2 \omega_{1}^{4}}{k_{1}^{\mathrm{p}} k_{3}^{\mathrm{p}}}, & \frac{\omega_{1}^{2} \omega_{5}^{2}}{4 k_{1}^{\mathrm{p}} k_{5}^{\mathrm{r}}} \\
\frac{\omega_{2}^{2} \omega_{6}^{2}}{2 k_{2}^{\mathrm{p}} k_{6}^{\mathrm{p}}}+\frac{\omega_{2}^{2} \omega_{5}^{2}}{k_{2}^{\mathrm{p}} k_{5}^{\mathrm{p}}}, & \frac{\omega_{2}^{4}}{k_{2}^{\mathrm{p}} k_{6}^{\mathrm{p}}}
\end{array}\right],} \\
& {\left[\Gamma^{\mathrm{rp}}\right]=\mu_{0}^{2} b^{2} \beta^{2}\left[\begin{array}{cc}
\frac{\omega_{1}^{2} \omega_{6}^{2}}{4 k_{1}^{\mathrm{r}} k_{6}^{\mathrm{p}}}+\frac{\omega_{1}^{2} \omega_{5}^{2}}{4 k_{1}^{\mathrm{r}} k_{5}^{\mathrm{p}}}, & \frac{\omega_{1}^{4}}{k_{1}^{\mathrm{r}} k_{6}^{\mathrm{r}}} \alpha^{2}(2 \alpha+\beta)^{2} \\
\frac{\omega_{2}^{2} \omega_{6}^{2}}{4 k_{2}^{\mathrm{k}} k_{6}^{\mathrm{p}}}+\frac{\omega_{2}^{2} \omega_{5}^{2}}{k_{2}^{k} k_{5}^{\mathrm{p}}}, & \frac{\omega_{2}^{4}}{k_{2}^{\mathrm{r}} k_{4}^{\mathrm{T}}}
\end{array}\right] \text {, }}
\end{aligned}
$$




$$
\begin{aligned}
& {\left[\Gamma^{\mathrm{rr}}\right]=\mu_{0}^{2} b^{2} \beta^{2}}
\end{aligned}
$$

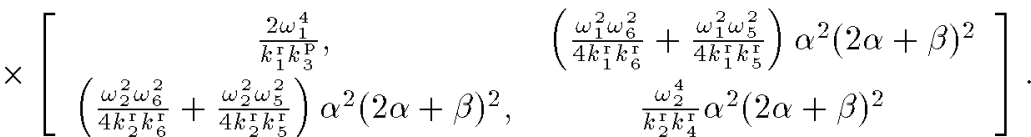

The right-hand side of Eq. (13) is - within the approximation of constant intensity - independent of space coordinate $s$, therefore phases of all waves are linear functions of $s$ :

$$
\Phi_{j}^{\mu}(s)=\eta_{j}^{\mu} s+\Phi_{j}^{\mu}(0)
$$

( $\mu=\mathrm{p}$ or $\mathrm{r}$ ). Under some natural conditions we may assume that initial values of all phases are equal to zero. This assumption was used in numerical calculations.

It is worthy stressing that coefficients $\eta$ and, consequently, also the phases $\Phi$ depend on intensities of the interacting waves (see Eq. (13)).

\section{Boundary conditions}

The continuity conditions for the left $(z=0)$ and right $(z=L)$ side of the plate involve two coefficients: the reflection $R$ and the transmission $T$. In general, they may depend both on the frequency and on the polarisation of the wave. For $s=0$ (that is $z=0$ ) we have

$$
T_{j}^{\mu} A_{j}^{\mu}(0-)+R_{j}^{\mu} B_{j}^{\mu}(0+)=A_{j}^{\mu}(0+) .
$$

For $z=L($ or $s=L / \alpha)$ we have

$$
R_{j}^{\mu} A_{j}^{\mu}(L-)=B_{j}^{\mu}(L-) \text {. }
$$

The symbol $B$ denotes the amplitude of the backward wave, equivalent of $A$. From the last equation it follows that

$$
\left|B_{j}^{\mu}\right|=\left|R_{j}^{\mu} \| A_{j}^{\mu}\right| .
$$

This relation holds for the whole plate. Therefore the change of the phase of backward waves (denoted as $\Psi$ ) inside the material is equal to

$$
\Delta \Psi_{j}^{\mu}=-\left|R_{j}^{\mu}\right| \Delta \Phi_{j}^{\mu} .
$$

The sign minus reflects the change of phase connected with the change of the direction of propagation.

The boundary condition leads to the final form for module of amplitudes

$$
\left|A_{j}^{\mu}\right|=\frac{T_{j}^{\mu} A_{0, j}^{\mu} \exp \left(\mathrm{i} \Delta \Phi_{j}^{\mu}\right)}{1-\left|R_{j}^{\mu}\right|^{2} \exp \left[\mathrm{i}\left(1-R_{j}^{\mu}\right) \Delta \Phi_{j}^{\mu}\right]}, \quad j=1,2, \quad \mu=\mathrm{p}, \mathrm{r} .
$$

Consequently, the equation for the transmitted intensities $I$ has the form

$$
I_{j}^{\mu}=\frac{\boldsymbol{T}_{j}^{\mu} I_{0, j}^{\mu}}{1+\left(\boldsymbol{R}_{j}^{\mu}\right)^{2}-2 \boldsymbol{R}_{j}^{\mu} \cos \left[\left(1-R_{j}^{\mu}\right) \Delta \Phi_{j}^{\mu}\right]},
$$

where we have introduced the intensity parameters $\boldsymbol{T}$ and $\boldsymbol{R}$, depending on the corresponding amplitude parameters $\left(T\right.$ and $R$ ) in the way: $\boldsymbol{T}=T^{2}, \boldsymbol{R}=R^{2}$.

The denominator of the expression on the right-hand side of Eq. (20) contains a periodic function whose argument is a monotonic function of intensities of the 
waves considered. Therefore we may expect a typical bistable behaviour for all components of the electric fields. This means that also the polarisation state will follow similar changes.

At the right side of the plate the electric vector takes on the value

$$
E_{j}^{\mu}=A_{j}^{\mu} \mathrm{e}^{-\mathrm{i} k_{j}^{\mu} L / \alpha} \text {. }
$$

These expressions may be next put in the coherence matrix $M$ for each frequency individually. The matrix is defined as follows [2]:

$$
\boldsymbol{M}_{j}=\left[\begin{array}{ll}
M_{x x}, & M_{x y} \\
M_{x y}, & M_{y y}
\end{array}\right]_{j}=\left[\begin{array}{cc}
E_{j}^{\mathrm{p}} \bar{E}_{j}^{\mathrm{p}}, & E_{j}^{\mathrm{p}} \bar{E}_{j}^{\mathrm{r}} \\
E_{j}^{\mathrm{p}} E_{j}^{\mathrm{r}}, & E_{j}^{\mathrm{r}} \bar{E}_{j}^{\mathrm{r}}
\end{array}\right] .
$$

We will limit ourselves to two fundamental waves $(j=1,2)$ as the secondary waves are of less importance for our analysis.

\section{Polarisation states}

The state of the polarisation of a wave characterised by the coherence matrix may be described by means of the polarisation ellipse. The ratio $b / a$ of its axes is equal to tangent of an angle $\theta$, which fulfils the relations

$$
\sin 2 \theta=\mathrm{i} \frac{M_{y x}-M_{x y}}{M_{x x}+M_{y y}}, \quad \tan \theta= \pm \frac{b}{a} .
$$

The angle $\psi$ between the main axis of the ellipse and the $O x$ axis is given by the formula

$$
\tan 2 \psi=\frac{M_{x y}+M_{y x}}{M_{x x}-M_{y y}} .
$$

After substituting (21) for $E$ and then (19) for amplitudes $A$ we obtain the following expressions:

$$
\begin{aligned}
& \sin 2 \theta=\frac{2 \sqrt{I^{\mathrm{r}} I^{\mathrm{p}}} \sin \left[\Phi^{\mathrm{r}}(L)-\Phi^{\mathrm{p}}(L)-\left(k^{\mathrm{r}}-k^{\mathrm{p}}\right) L / \alpha\right]}{I^{\mathrm{r}}+I^{\mathrm{p}}}, \\
& \tan 2 \psi=\frac{2 \sqrt{I^{\mathrm{r}} I^{\mathrm{p}}} \cos \left[\Phi^{\mathrm{r}}(L)-\Psi^{\mathrm{p}}(L)-\left(k^{\mathrm{r}}-k^{\mathrm{p}}\right) L / \alpha\right]}{I^{\mathrm{r}}-I^{\mathrm{p}}} .
\end{aligned}
$$

As the phases $\Phi$ depend monotonically on intensities $I$ in a manner which in general allows the bistable behaviour, the same may be expected for the parameters characterising the polarisation ellipse.

\section{Numerical calculations}

In order to simplify numerical calculations we assumed that both incident beams are polarised linearly; the beam with the frequency $\omega_{1}$ had two components: one parallel and one perpendicular to the incident plane whereas the second fundamental wave (with the frequency $\omega_{2}$ ) had only one component - that parallel to this plane. We have calculated intensities and polarisation state of the first wave 
in dependence on intensities of incoming waves and on different phenomenological parameters.

1. The dependence of the transmitted wave (with $\omega=\omega_{1}$ ) characteristics on the relative intensity $I_{1}$ (normalised to the incident beam) of the first beam is shown in Figs. 1a-d and Figs. 2a, b. The first of them (1a) shows the behaviour of phase of one of the three components. Next three plots (1b, c, d) show the intensity of the normal component $\left(I_{1 \mathrm{p}}\right)$ and intensities of parallel components $I_{1 \mathrm{r}}$
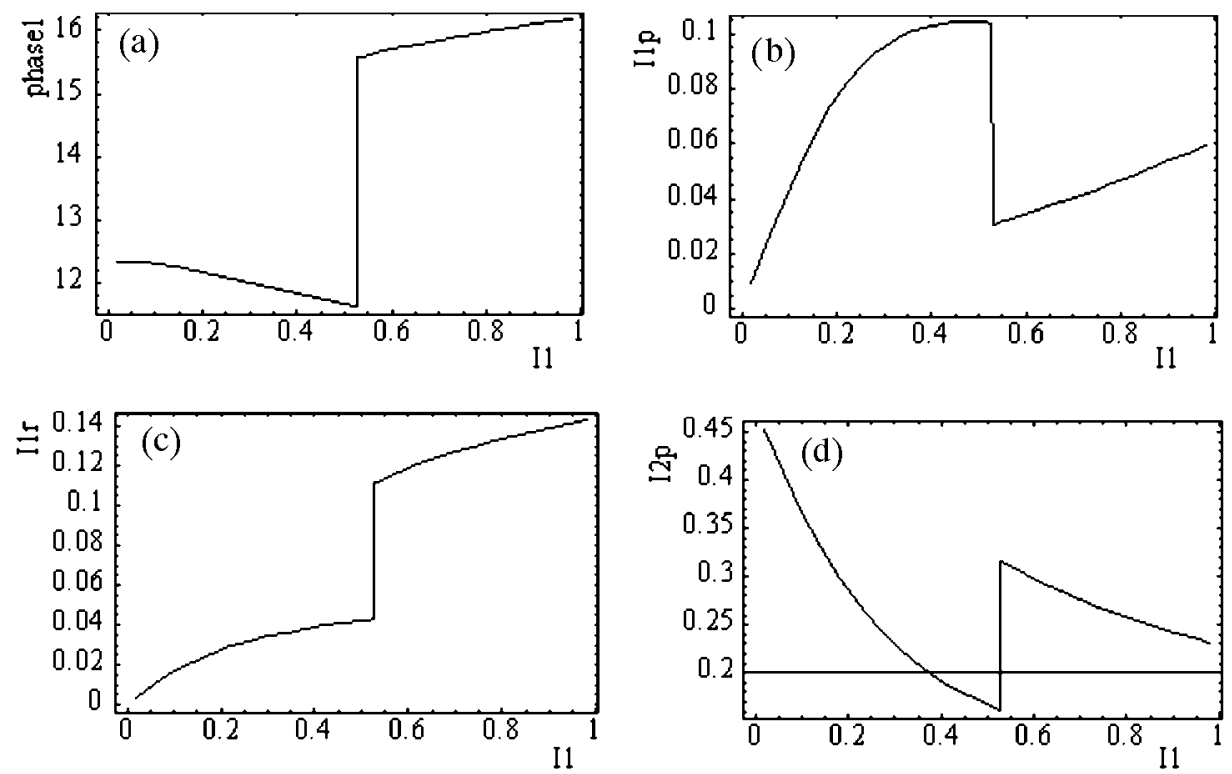

Fig. 1. The dependence of the phase (a) and intensity of the first component of the first wave (b), the intensity of the second component of the first wave (c) and intensity of the second wave (d) on the total intensity $I_{1}$ of the first wave on the entrance. All intensities are normalised to the incoming intensities of the corresponding two waves.
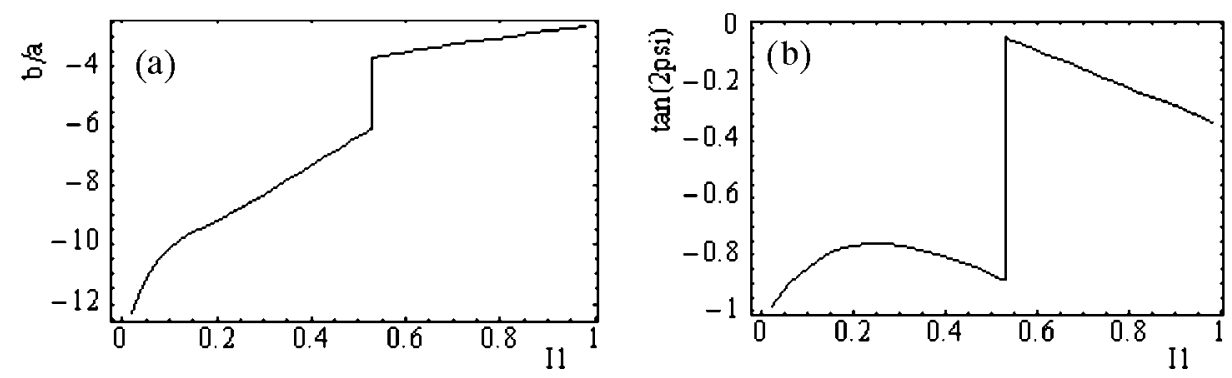

Fig. 2. The dependence of two parameters of polarisation ellipse: the ratio $b / a$ of axes (a) and the angle of inclination (b) of the first wave on the initial intensity $I_{1}$ of the same wave. 
and $I_{2 \mathrm{r}}$. Finally, we present the ratio $b / a$ of the axes of polarisation ellipse of the first wave (Fig. 2a) and tangent of the (doubled) angle of inclination of the main axis (b) of this ellipse (Fig. 2b). In all cases we have assumed the following values of the parameters: amplitude reflection coefficient $R=0.7, \alpha=0.5$ and relative refraction index $n_{\mathrm{r}} / n_{\mathrm{p}}=1.3$. Nonlinear susceptibility parameter is combined with length $L$ and the reversed linear refraction index $n_{\mathrm{p}}$. Their product (together with some other constants) was chosen to be 97.55 . The shape of all curves appeared to be very sensitive to its value; we have chosen the most regular ones among those exhibiting the bistable behaviour. It is easy to see that at a certain value of the intensity of the first wave (with frequency $\omega_{1}$ ) all the quantities considered undergo a jump which may correspond to a bistable character of this dependence.

2. Similar behaviour was observed when we allowed to change $I_{2}$ instead of $I_{1}$ (the latter was kept constant at its maximal value). In Figs. 3a,b we have
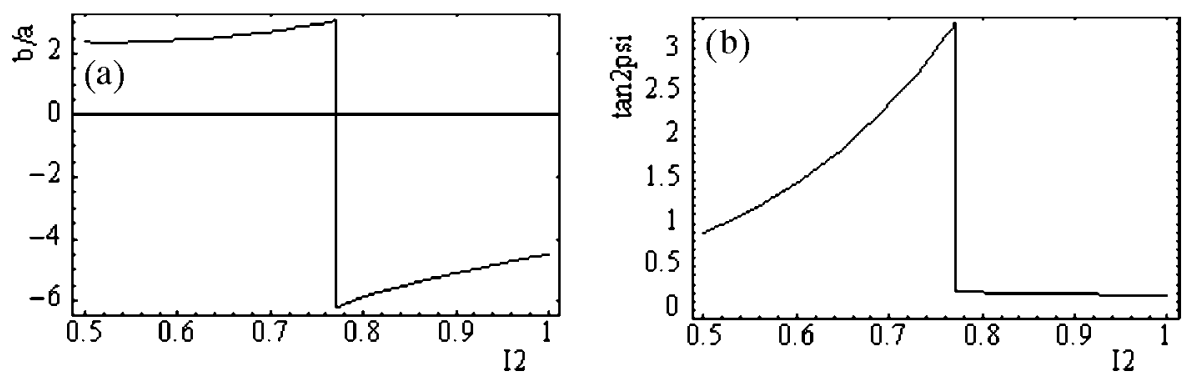

Fig. 3. The dependence of two parameters of polarisation ellipse: the ratio $b / a$ of axes (a) and the angle of inclination (b) of the first wave on the output on the initial intensity $I_{2}$ of second wave.

depicted two ellipse parameters (the ratio of axes $b / a$ and $\tan (2 \psi)$ ). This time the ratio $b / a$ changes its sign when passing through a value of $I_{2}$ which means the change of the direction of rotation of the vector $\boldsymbol{E}$ over the ellipse.

3. Another interesting property is connected with the angle of polarisation of the incident wave $\omega_{1}$. In the following figures the variable $\alpha$ denotes the relative
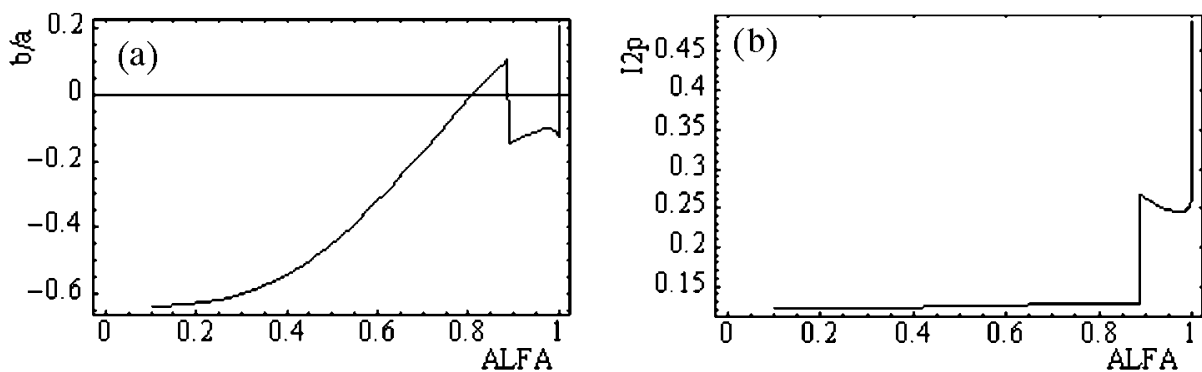

Fig. 4. The dependence of the ratio $b / a$ on the direction of (linear) polarisation of the same first wave on the input. Part (b) shows how this dependence manifests in the behaviour of the intensity of the second beam. 
percentage of the normal component to the parallel one in the incident beam. The polarisation state of the transmitted wave is illustrated in Fig. 4a. The intensity of the second beam behaves as in Fig. 4b. It is easy to see that the angle of polarisation of the incident beam may significantly influence the elliptic polarisation of the outgoing beam leading to discontinuity at a specific value of this angle.

4. Similar effects were noticed if we changed the anisotropy of the medium. As its measure we have used the ratio of refractive indices corresponding to two mutually perpendicular polarisations, e.g. $n_{\mathrm{r}} / n_{\mathrm{p}}$. In Figs. $5 \mathrm{a}$, b this ratio is denoted
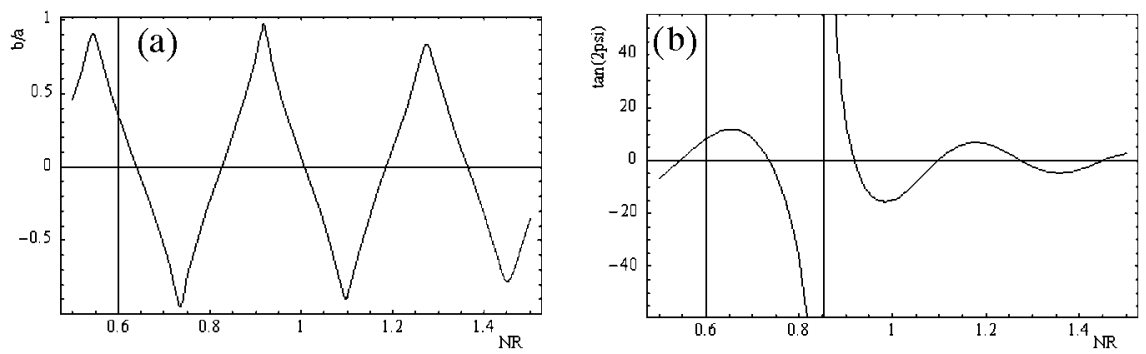

Fig. 5. The dependence of $b / a(a)$ and $\tan (2 \psi)$ (b) on the anisotropy of the medium.

by NR. As in previous figures we limit here to the case of polarisation ellipse parameters. The most interesting to us is the behaviour of the ratio $b / a$. By changing NR we may reduce the ellipse to a line $(b / a=0)$ and then to convert it into a circle $(b / a=1)$. This process repeats successively.

\section{Summary}

Interaction of two incident monochromatic beams of similar power via a nonlinear and anisotropic medium may lead to significant modifications of these beams. Both intensity and polarisation state may exhibit bistable behaviour. The underlying mechanism is based on the second-order nonlinearity which produces appropriate changes of phase of all waves involved in this process. They result in changes of intensities and, consequently, changes of the polarisation state. For some values of parameters these changes may be drastic as seen from presented figures. In some cases one may observe a bistable behaviour.

The performed numerical calculations are only qualitative and the choice of values of physical parameters is to some extent arbitrary but not unrealistic. We hope that the model may be easily adopted to real situations.

\section{References}

[1] R.W. Boyd, Nonlinear Optics, Academic Press, Boston 1992.

[2] I. Buchvarov, S. Saltiel, Ch. Iglev, K. Koynov, Opt. Commun. 141, 173 (1997).

[3] V. Leutheuser, U. Trutschel, F. Lederer, J. Opt. Soc. Am. B 9, 1145 (1995).

[4] L.C. Khoo, Y. Liang, H. Li, IEEE J. Quantum Electron. 28, 1816 (1992).

[5] W. Jęda, A. Zagórski, Acta Phys. Pol. A 96, 373 (1996). 